\title{
Implementation of multistrip crystals to protect the septum magnets and to generate gamma radiation
}

\author{
A. G. Afonin, E. V. Barnov, G. I. Britvich, Yu. A. Chesnokov, P. N. Chirkov, A. A. Durum, \\ M. Yu. Kostin, I. S. Lobanov, V. A. Maisheev, I. V. Poluektov, S. F. Reshetnikov, \\ Yu. E. Sandomirskiy, D. A. Savin, and A. A. Yanovich \\ NRC Kurchatov Institute-IHEP, 142281 Protvino, Russia
}

(Received 10 December 2018; published 19 March 2019)

\begin{abstract}
Recently studies are started on the application of volume reflection of particles in crystals for the steering beams (for extraction and collimation of a circulating beam in accelerators). Volume reflection is more efficient than channeling, but requires amplification of the deflection angle by applying multicrystals. The report discusses two new applications of multicrystals made like multistrip structures: (i) the property of effective deflection of particle beam was used to protect the septum magnets of the U-70 in the process of extraction of the proton beam with energy of $50 \mathrm{GeV}$; (ii) the possibility of generation of gamma radiation was studied in the secondary electron beam with energy of $7 \mathrm{GeV}$. In both cases, promising preliminary data were obtained.
\end{abstract}

DOI: 10.1103/PhysRevAccelBeams.22.033001

\section{INTRODUCTION}

In the early 1950s, Ter-Mikaelyan and Uberall [1,2] theoretically showed that in an ordered medium such as single crystals the bremsstrahlung of electrons and positrons of high energy has an interference nature and differs from their radiation in amorphous media. Later experiments confirmed these theoretical predictions. Since then, this radiation has been investigated theoretically in many papers and tested experimentally under various conditions such as beam energy and various beam orientations and crystal matter.

In particular, it was shown that the nature of the radiation process changes with increasing electron energy and the radiation becomes more similar to magnetic bremsstrahlung. The proposal of Tsyganov [3] to use bent single crystals to deflect positively charged particles was important in the physics of the interaction of charged particles with single crystals. It turned out that the processes of interaction of particles with bent single crystals differ from processes in straight crystals. Thus, the so-called phenomenon of volume reflection $[4,5]$ of a particle beam from bent crystallographic planes was discovered. In addition, the radiation of electrons and positrons in the plane fields of bent single crystals was predicted [6] and then measured [7], which accompanies the process of volume reflection.

Published by the American Physical Society under the terms of the Creative Commons Attribution 4.0 International license. Further distribution of this work must maintain attribution to the author(s) and the published article's title, journal citation, and DOI.
These two phenomena were the basis for our experimental study.

The volume reflection effect was found in Monte Carlo calculations of the motion of positively and negatively charged particles in bent single crystals [4]. An analytical description of this process can be found in $[5,8]$. In the experiments a very good agreement between the theoretical description and the measurement results was found [9]. The physics of the process consists in the fact that at certain angles of entry of particles into the crystal with respect to the crystallographic planes, these particles are reflected from these planes. However, even under optimal conditions, the mean angle of volume reflection does not exceed 1.5 critical channeling angles (i.e., relatively small). Despite this fact, the process of volume reflection is of some interest because it has a high efficiency of reflection. In other words, with volume reflection, almost all the particles are reflected.

When the reflected particle moves, its transverse coordinate in the plane of the bend undergoes aperiodic oscillations. Due to this character of motion, the electron (positron) emits gamma quanta. A theoretical description of this radiation process can be found in the papers [10-12]. The first experiment to detect such radiation was performed at electron energy of $10 \mathrm{GeV}$ [13] and showed satisfactory agreement with the calculations. Then, measurements of the radiation with volume reflection in silicon crystals at energies 180 [14] and 120 [15] GeV were performed. The results of the measurements were in good agreement with the calculations.

In the experiment [15], measurements of the radiation energy losses during channeling and volume reflection of $120 \mathrm{GeV}$ positrons were performed on the same crystal with 
a thickness of about $2 \mathrm{~mm}$ and with the same bending radii. A comparison of these energy losses with each other shows that for sufficiently large bending radii they are approximately the same. However, the intensity of the radiation (the number of emitted photons per unit length) is greater for channeled positrons. This is equivalent to the fact that in channeling more photons are emitted (per unit length) than with volume reflection and they have less energy.

The situation for electrons is essentially different. As is known, electrons captured in planar crystallographic channels are rapidly dechanneled. Because of this, radiation in volume reflection in bent crystals (and a thickness of $1 \mathrm{~mm}$ or more) is the main source of radiation losses of electrons. We note that the radiation in bent planes and in straight (unbent) planes of single crystals differs among themselves, although there is a definite relationship between them (see $[10,11])$. In comparison with radiation in bent planes, the radiation of electrons in straight planes has a sharper dependence on the angle of entry of particles into a single crystal relative to its planes and the region of such angles is much smaller. This circumstance can play an important role in applications such as collimation of beams since a collimated electron beam can have a noticeable angular divergence. In fact, in order to get into the channeling regime, a high-energy particle must get into the crystal within the critical channeling angle. This angle is always less than $0.1 \mathrm{mrad}$ at energies above $5-10 \mathrm{GeV}$. The region of angles for effective volume reflection is $d / R$, where $\mathrm{d}$ is the thickness of the crystal along the beam motion and $\mathrm{R}$ is the bending radius of the crystal. In addition, with volume reflection, the efficiency of this process is close to 1 [16], while in channeling an appreciable part of the particles is lost due to dechanneling, especially at low particle energies. However, the shortcoming of the volume reflection process is that the reflection angle is small and does not exceed 1.5 and 0.8 from the critical channeling angle, respectively, for positive and negative particles [9].

In this regard, it was proposed to use multistrip systems consisting of a series of identical striped bent single crystals $[17,18]$. Experiments showed the operability of this idea. Of course, the resulting angle in such systems is generally somewhat less than the expected angle calculated as the product of a single angle by the number of strips. Such systems were created not only for the planar of a case but also for the axial one. In this connection, the question arises as to the behavior of the radiation losses of energy of highenergy electrons or positrons in multistrip systems.

The present experiment is devoted to the study of scattering (volume reflection) of $50 \mathrm{GeV}$ protons and radiation losses of energy by $7 \mathrm{GeV}$ electrons in a multistrip crystal deflector.

\section{MULTISRIP CRYSTAL DEFLECTOR}

Figure 1 shows the appearance of the prepared multistrip crystal device. The device comprises 24 bent crystals in the (a)
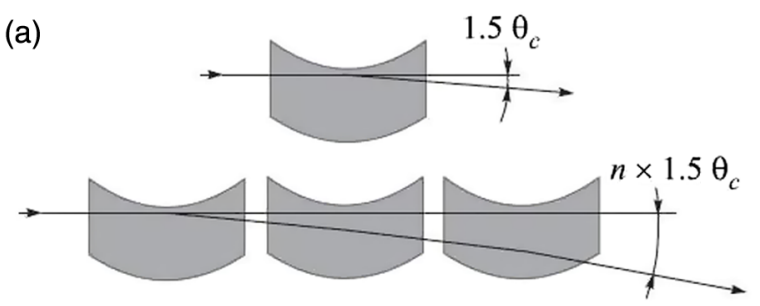

(b)

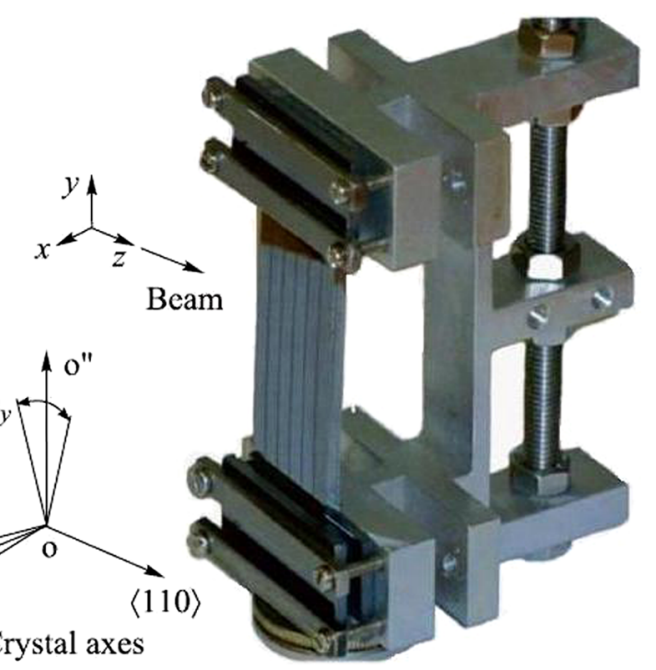

FIG. 1. Multilayer crystal radiator for the beam: (a) the schematic of operation and (b) the appearance and arrangement with respect to the beam.

form of bent strips. The method of bending several strips in one holder was developed in experiments on volume reflection of proton and negative pion beams [18]. Six strips along a beam of $2.5 \mathrm{~mm}$ each reinforce the radiation effect, and four layers of $0.5 \mathrm{~mm}$ across the beam provide the necessary transverse dimension of $2 \mathrm{~mm}$ (thick crystals cannot bend to the required angles, so a sandwich is needed). After optical verification, the device was placed in a two-axis goniometer. The bending angle of each strip is $1.1 \mathrm{mrad}$. It is larger than the Coulomb scattering angle of electrons with energy of $7 \mathrm{GeV}$ on a chain of six crystals, which is equal to $0.8 \mathrm{mrad}$.

For each individual strip, its characteristics for deviation and ability to radiation were calculated. It should be noted that the existing mathematical apparatus allows us to calculate these characteristics fairly accurately (see $[9,16]$, where calculations and measurements are compared).

So according to these calculations, the average reflection angle of $50 \mathrm{GeV}$ of the proton beam for the $2.5 \mathrm{~mm}$ silicon strip [in the (111) plane] is $38 \mu \mathrm{rad}$, and for $7 \mathrm{GeV}$ electrons $81 \mu \mathrm{rad}$. We also calculated radiation losses of $7 \mathrm{GeV}$ electrons in the plane fields (111) of a bent silicon single crystal (see Fig. 2).

The calculation was performed for two different thicknesses along the beam equal to 2.5 and 15 millimeters. In fact, energy losses for radiation in bent and straight single crystals represent the sum of two terms of the coherent and 


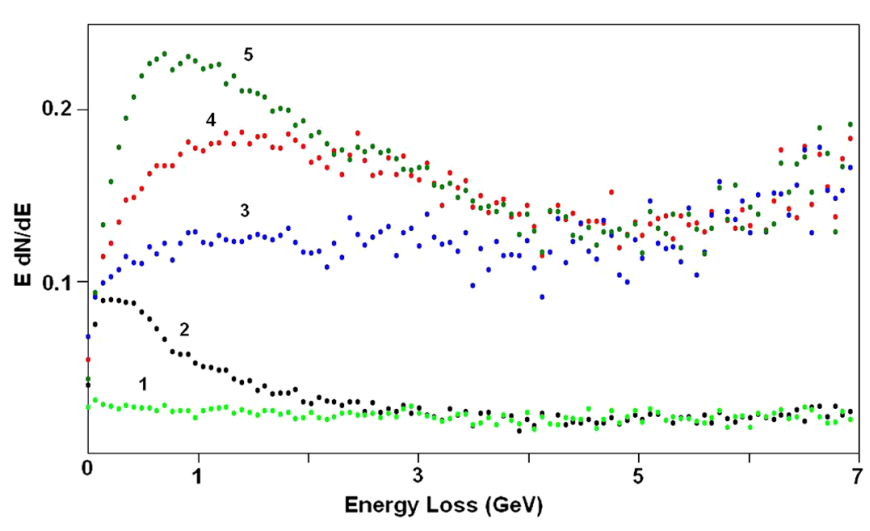

FIG. 2. The calculated energy losses: (1) in one 2.5 nonoriented strip; (2) in one strip oriented in planar orientation; (3) in nonoriented multistrip (see Fig. 1); (4) in single crystal (oriented in planar orientation) with the thickness $15 \mathrm{~mm}$; (5) multistrip in planar orientation.

incoherent [1,2,19]. The coherent term is due to the interaction with the electromagnetic field of the crystal, while the incoherent term is due to the interaction with the individual atoms of a crystal. Moreover, the incoherent term does not depend on the orientation of the beam relative to the crystallographic axes. We can assume that this term is approximately equal to the energy loss in amorphous silicon.

In Fig. 2 curves 1 and 2 illustrate the energy losses for radiation in a silicon crystal $2.5 \mathrm{~mm}$ long (equal to the length of one single strip, see Fig. 1) for nonoriented and oriented cases, respectively. Curves 3 and 4 show the same corresponding energy losses, but for a single crystal with a length equal to $6 \times 2.5=15 \mathrm{~mm}$. Curve 5 corresponds to the calculation for an oriented multistrip with six strips. Note that curve 4 describes the last case, of course. The calculations were performed by the Monte Carlo method. Thus, the calculated total energy losses (integrated over energy) are equal to $0.158,0.229,0.878,1.072$ and $1.148 \mathrm{GeV}$ for the curves $1-5$, correspondingly. In the accordance of these calculations the radiation energy losses are mostly large in the multistrip crystal. The axial orientation of the multistrip relative to electron beam motion is more difficult for calculations. However we expect the similar effect in this case but more strong.

\section{EXPERIMENTAL SETUP}

The experiment was performed in the $4 \mathrm{a}$ beam line of the U70 accelerator (Fig. 3). The $7-\mathrm{GeV}$ electron beam with an intensity of $\sim 10^{4}$ per cycle was guided to the crystal device placed in the goniometer. The step of the horizontal and vertical rotation of the goniometer was $0.02 \mathrm{mrad}$. A telescope of scintillation counters $S_{1}, S_{2}$, and $S_{3}$ separated a fraction of particles entering the crystal radiator and formed an angular divergence of $\sigma_{x} \sim 0.5 \mathrm{mrad}$ and $\sigma_{y} \sim 1 \mathrm{mrad}$. The last counter had a $2 \times 30-\mathrm{mm}$ cross

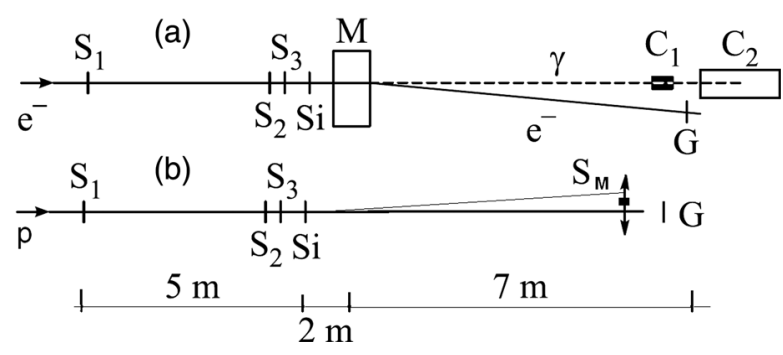

FIG. 3. Schematic of the crystal setup: $\left(S_{1}-S_{3}\right)$ scintillation counters, (G) hodoscope for the determination of particle coordinates, $(\mathrm{Si})$ crystal in the goniometer. (a) For electron beam: $\left(C_{1}, C_{2}\right)$ calorimeters for the determination of the energy of photons, (M) magnet. (b) For proton beam: $\left(S_{M}\right)$ the scanning counter.

section, coinciding with the cross section of the crystal assembly; it was mounted on the end of the radiator and could be displaced with it in the goniometer owing to a fiber optic connection to a photomultiplier. A vertically deflecting magnet $\mathrm{M}$ with a magnetic-path length $\mathrm{Bl}=0.33 \mathrm{~T} \times \mathrm{m}$ separated the emitted photons and interacting electrons. A 5-mm-step scintillation hodoscope with the recording of information on the hodoscope photomultiplier could spectroscopically measure the energy of electrons. The energy of photons was measured by a lead glass calorimeter $C_{2} 20$ radiation lengths long. A miniature calorimeter $C_{1}$ with transverse dimensions of $10 \mathrm{~mm} \times 20 \mathrm{~mm}$ and a length of $7 \mathrm{~mm}$ made of $\mathrm{CeF}_{3}$ heavy scintillator was used, as the first step of detection (so-called preshower detector) for the rejection of the background. For the arms of increasing of the efficiency of detection of photons the $5 \mathrm{~mm}$ tungsten converter $C_{w}$ (standing before $C_{1}$ ) was used in some measurements. In addition, it was used for the fast adjustment of the planar and axial orientations of the crystal target. First, the principal (111) plane was determined, along which silicon strips were cut and bent, by varying the horizontal angle $\Phi_{x}$. Then, the horizontal angle was fixed in the optimal position and the vertical angle $\Phi_{y}$ was varied in order to find the axial effect. Figure 4 shows the dependences of the count rate $C_{1}$ on the horizontal and vertical angles of the goniometer. The spectra of photons, emitted energy, and energy loss of electrons were measured for three orientations of the goniometer: planar orientation, axial orientation, and disoriented state of the crystal target.

The experimental setup was insignificantly changed for measurements with the use of $50 \mathrm{GeV}$ proton beam. The magnet $M$ was switched off and electromagnetic calorimeters were removed from the beam line [see Fig. 3(b)]. The special scanning scintillator counter (placed on a distance 7 meters after the multistrip crystal) was used for measurements of beam profiles in the horizontal plane. The proton beam was extracted from the accelerator by means of a silicon crystal deflector (with the bending angle equal to $89 \mathrm{mrad}$ ) installed in the vacuum chamber (in 27 accelerator block). We used in measurements the relatively small intensity equal to $10^{5}$ protons per cycle. 

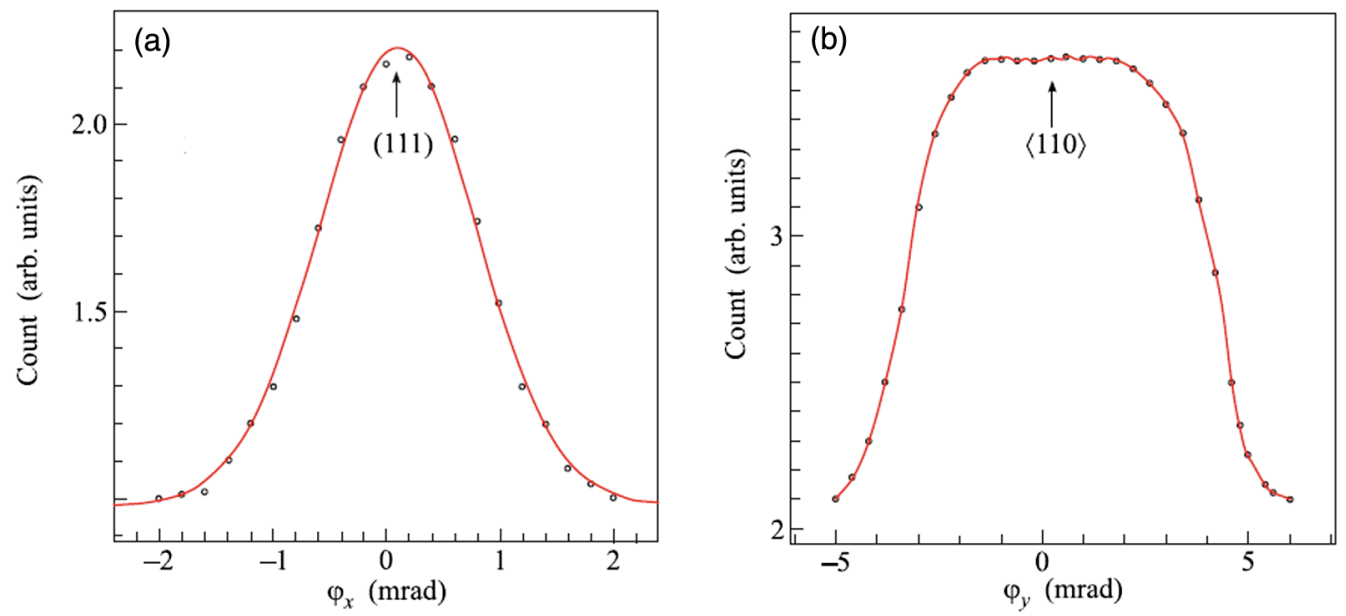

FIG. 4. Count rate of the calorimeter $C_{1}$ versus the (a) horizontal and (b) vertical angles.

The coordinate sizes (horizontal and vertical) of proton beam were about $1 \mathrm{~cm}$ on the crystal device.

\section{THE EXPERIMENTAL RESULTS}

\section{A. Measurement of the radiation energy losses of $7 \mathrm{GeV}$ electrons}

The value of radiation energy losses of beam of the $7 \mathrm{GeV}$ electrons in multistrip crystal was investigated as a function of energy for the tree orientations. In the experiment the value $T_{1} / T_{0}$ was measured, where $T_{1}$ is the coincidence of counters $S_{1}, S_{2}, S_{3}, C_{1}, C_{2}$ and $T_{0}$ is coincidence of counters $S_{1}, S_{2}, S_{3}$. The energy loss for every event (defined by the relation $T_{1} / T_{0}$ ) was determined with the help of the $C_{2}$ calorimeter.

It is easy to see that $T_{0}$ is the number of electrons passed through the multistrip and $S_{1}, S_{2}, S_{3}$ counters. In the multistrip, some part of the electrons emit photons which register with the help of $C_{1}$ and $C_{2}$ calorimeters. Then all electrons are deflected by the $\mathrm{M}$ magnet. However, some part of the emitted photons moving from the multistrip into $C_{1}$-calorimeter are lost due to geometrical reasons. Taking into account that for photon energies more than $0.2 \mathrm{GeV}$ [20] the efficiency of registration of photons by the $C_{1}$ calorimeter is independent of their energy. Because of this, part of the photon losses is defined by geometrical parameters of electron beam and is independent of energy. Thus, the value $T_{1} / T_{0}$ determines the form of the distribution of the radiation energy losses.

The first preliminary results of this experiment were published in the paper [21]. Here the measured quantities of radiation energy losses were presented in relative units. More informative data would exist if it were possible to present these data as a probability density function per one electron passed through a multistrip. In the present paper we obtain such a presentation of experimental data. This result is based on calculation of radiation energy losses in the disoriented single crystal. Such energy losses are similar to radiation energy losses in an amorphous medium. These losses are well known and hence we can obtain the reliable result for nonoriented single crystal.

The results of measurements of radiation energy losses of $7 \mathrm{GeV}$ electrons in natural (absolute) scale are presented in Fig. 5. It should be noted that during measurements the channels in the calorimeter $C_{2}$ were saturated near the end of the spectrum and thus measurements were possible only up to the energy of radiation losses equal to $5 \mathrm{GeV}$. However, from theoretical estimations and from Fig. 6 one can see that at energy losses more than $5 \mathrm{GeV}$ all the curves have approximately the same meaning. From Fig. 5 we see that the form of experimental curve for nonoriented crystal is in a good agreement with the theoretical calculation. From here we find the absolute scale of radiation losses of energy. The small difference between the curves (experimental and theoretical for nonoriented crystal) at small energies $(<1 \mathrm{GeV})$ can be explained by more wide propagation of electromagnetic shower in transverse space in comparing with more energy losses. According to our estimation the influence of energy resolution of calorimeter on the form of curve is weak. Thus we can find the mean radiation energy losses for various orientation of crystal. They are $0.889 \mathrm{GeV}$ for the curve $1,1.19 \mathrm{GeV}$ for curve 2 and $2.15 \mathrm{GeV}$ for curve 3 .

\section{B. Reflection of the $50 \mathrm{GeV}$ proton beam}

The same crystal device was used for the effective deflection of the proton beam due to the reflection phenomenon [16.]. Volume reflection is more efficient than channeling, but requires amplification of the deflection angle by applying multicrystals as shown in Fig. 1. The horizontal coordinate distributions of $50 \mathrm{GeV}$ proton beam were measured with the help of scanning counter for two orientations: (a) disoriented and (b) oriented in the maximum of planar volume reflection. Figure 6(a) 


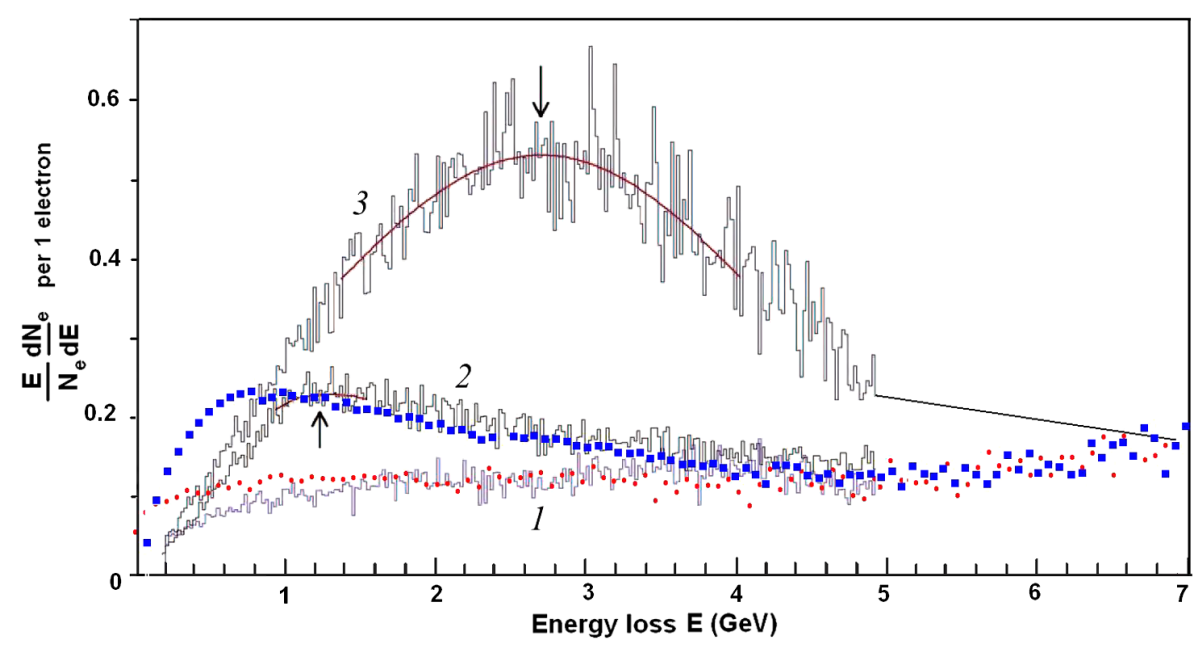

FIG. 5. Measured distributions of energy losses of $7 \mathrm{GeV}$ electrons in the multistrip device: the curves 1,2 and 3 are for the cases of nonoriented, planar orientation and axial orientation, correspondingly. The red points and blue squares are calculation for nonoriented and planar oriented cases.
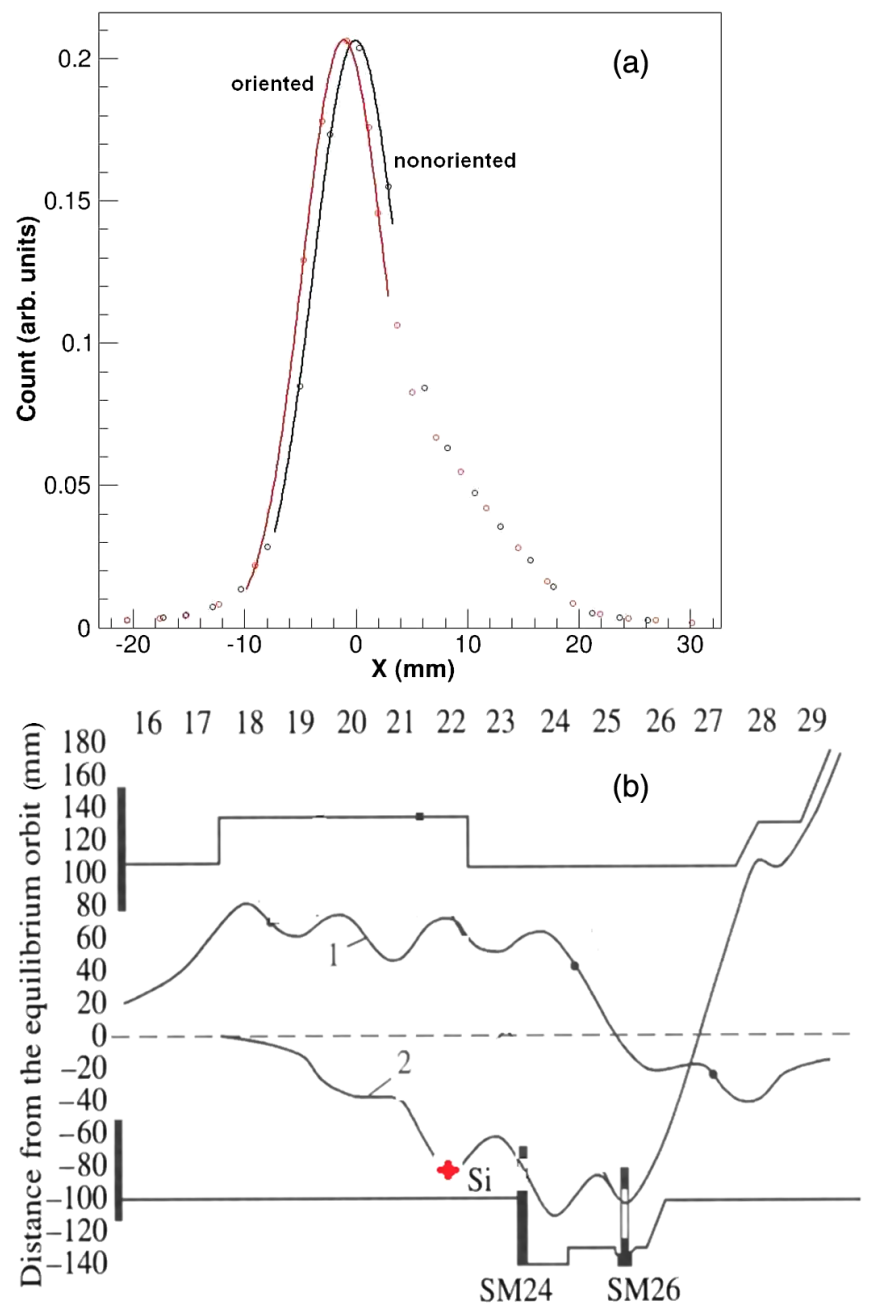

1 - circulating beam, 2 - extracted beam

FIG. 6. (a) The beam profiles in orientation and nonoriented cases. (b) The scheme of SM24 septum shadowing in the U70 ring. illustrates the results of these measurements. For every orientation the coordinate of the center of distribution was found. Accordingly to measurements the difference of distribution centers was equal to $1.1 \mathrm{~mm}$. It is easy to see that such value of displacement corresponds to deflection angle of the beam equal to $0.16 \mathrm{mrad}$. The maximal theoretical value of this angle is equal to $0.038 \times 6=0.228 \mathrm{mrad}$. Thus we can conclude about satisfactory agreement between experiment and theoretical expectation. The next step is to create a station with this crystal in the 22 block of the U70 and implement the experience to protect the SM24 septum in the 24 accelerator block, as shown in Fig. 6(b). The magnet SM24 has 2-mm septum width, and according to our estimates, the crystal should have a good shadow (see also [22]).

\section{CONCLUSION}

In the presented paper the interaction of high energy particles with a multistrip crystal device was investigated experimentally. It was demonstrated that observed in measurements effects (as reflection and radiation power) increase in this device. The measured increasing is less than multiplication of the number strips on the value for one strip. For reflection this result was considered previously in the corresponding section. For radiation it also follows from our measurements. So, for one strip the calculated value of energy losses for planar orientation is equal to $0.229 \mathrm{GeV}$. The multiplication is $6 \times 0.229=1.374$. This is not much more than measured value equal to $1.19 \mathrm{GeV}$.

We propose to use the reflection of proton beam in multistrip for protection of septum magnets on accelerators and for collimations of electron, positron and high energy photon beams on the future electron-positron colliders. In particular, we plan such work on the accelerator U-70. 


\section{ACKNOWLEDGMENTS}

The work is supported by Russian Science Foundation (Grant No. 17-12-01532).

[1] M. L. Ter-Mikaelian, High Energy Electromagnetic Processes in Condensed Media (Wiley, New York, 1972).

[2] H. Uberall, Phys. Rev. 103, 1055 (1956); 107, 223 (1957).

[3] E. N. Tsyganov, Report No. FERMILAB-TM-0682, Batavia, 1976.

[4] A. M. Taratin and S. A. Vorobiev, Volume reflection" of high-energy charged particles in quasi-channeling states in bent crystals, Phys. Lett. A 119, 425 (1987).

[5] V. A. Maisheev, Volume reflection of ultrarelativistic particles in single crystals, Phys. Rev. ST Accel. Beams 10, 084701 (2007).

[6] Yu. A. Chesnokov, V. I. Kotov, V. A. Maisheev, and I. A. Yazynin, Radiation of photons in process of charged particle volume reflection in bent monocrystal, J. Instrum. 3, P02005 (2008).

[7] W. Scandale et al., Experimental study of the radiation emitted by $180 \mathrm{GeV} / c$ electrons and positrons volume reflected in a bent crystal, Phys. Rev. A 79, 012903 (2009).

[8] M. V. Bondarenco, Model solution for volume reflection of relativistic particles in a bent crystal, Phys. Rev. A 82, 042902 (2010); N. Shul'ga, V. Truten', V. Boiko, and A. Esaulov, Scattering of high energy particles by field of the bent atomic planes, Phys. Lett. A 376, 1617 (2012).

[9] S. Bellucci, Y. A. Chesnokov, V. A. Maisheev, and I. A. Yazynin, Volume reflection and volume capture of ultrarelativistic particles in bent single crystals, Phys. Rev. ST Accel. Beams 18, 114701 (2015).

[10] V. A. Maisheev, Photon emission and photoproduction processes in bent single crystals, Nuovo Cimento C 34, 73 (2011).

[11] S. Bellucci and V. A. Maisheev, Photon emission and electron-positron photoproduction processes in the planar field of a bent single crystal, Phys. Rev. A 86, 042902 (2012).
[12] V. Guidi, L. Bandiera, and V. Tikhomirov, Radiation generated by single and multiple volume reflection of ultrarelativistic electrons and positrons in bent crystals, Phys. Rev. A 86, 042903 (2012).

[13] A. G. Afonin et al., Investigation of the emission of photons induced in the volume reflection of $10-\mathrm{GeV}$ positrons in a bent silicon single crystal, JETP Lett. 88, 414 (2008).

[14] W. Scandale et al., Experimental study of the radiation emitted by $180-\mathrm{GeV} / c$ electrons and positrons volumereflected in a bent crystal, Phys. Rev. A 79, 012903 (2009).

[15] D. Lietti et al., Radiation emission phenomena in bent silicon crystals: Theoretical and experimental studies with $120 \mathrm{GeV} / c$ positrons, Nucl. Instrum. Methods Phys. Res., Sect. B 283, 84 (2012).

[16] W. Scandale et al., Volume reflection dependence of $400-\mathrm{GeV} / \mathrm{c}$ protons on the bent crystal curvature, Phys. Rev. Lett. 101, 234801 (2008).

[17] W. Scandale et al., Multiple volume reflections of highenergy protons in a sequence of bent silicon crystals assisted by volume capture, Phys. Lett. B 688, 284 (2010).

[18] W. Scandale et al., Comparative results on the deflection of positively and negatively charged particles by multiple volume reflections in a multi-strip silicon deflector, JETP Lett. 101, 679 (2015).

[19] S. Bellucci and V. A. Maisheev, Coherent bremsstrahlung in imperfect periodic atomic structures, Phys. Rev. B 71, 174105 (2005).

[20] J. H. Hubbell, Photon cross sections, attenuation coefficients, from $10 \mathrm{KeV}$ to $100 \mathrm{GeV}$, NSRDS-NBS-29.

[21] A. G. Afonin et al., Emission of photons at the interaction of a high-energy electron beam with a sequence of bent single crystals, JETP Lett. 107, 451 (2018).

[22] F. M. Velotti, M. A. Fraser, B. Goddar, V. Kain, W. Scandale, and L. S. Stoel, Reduction of resonant slow extraction losses with shadowing of septum wires by a bent crystal, in Proceedings of IPAC2017, Copenhagen, Denmark, http:// accelconf.web.cern.ch/AccelConf/ipac2017/papers/ mopik050.pdf. 